Pacific

Journal of

Mathematics

MODULAR DIOPHANTINE INEQUALITIES AND NUMERICAL SEMIGROUPS

J. C. Rosales, P. A. García-SÁnchez and J. M. Urbano-Blanco 


\title{
MODULAR DIOPHANTINE INEQUALITIES AND NUMERICAL SEMIGROUPS
}

\author{
J. C. Rosales, P. A. García-Sánchez and J. M. Urbano-Blanco
}

\begin{abstract}
We study the set of integer solutions to the modular diophantine inequality $a x \bmod b \leq x$.
\end{abstract}

\section{Introduction}

Given $x \in \mathbb{Q}$, we set $\lceil x\rceil=\min \{z \in \mathbb{Z} \mid z \geq x\}$ and $\lfloor x\rfloor=\max \{z \in \mathbb{Z} \mid z \leq x\}$, as usual. Given integers $m, n$ with $n>0$, we set $m \bmod n=m-n\lfloor m / n\rfloor$ and $m \bmod (-n)=m \bmod n$. A modular diophantine inequality is an expression of the form $a x \bmod b \leq x$ with $a, b$ integers such that $b \neq 0$. Since $a x \bmod b \geq 0$, the set $S$ of solutions to such an inequality is contained in the set $\mathbb{N}$ of nonnegative integers. $S$ is a numerical semigroup, that is, $S$ is closed under addition, $0 \in S$ and $\mathbb{N} \backslash S$ is finite. Not every numerical semigroup arises from a modular diophantine inequality, and Section 2 presents a procedure for testing numerical semigroups for this property. Theorem 12 is crucial for obtaining this algorithm, and thus Section 1 is devoted to it. One of the main consequences of this theorem is that if the inequalities $a x \bmod b \leq x$ and $c x \bmod d \leq x$ have the same solutions, then

$$
b-(a, b)-(a-1, b)=d-(c, d)-(c-1, d),
$$

where $(x, y)$ denotes the greatest common divisor of the integers $x$ and $y$.

A numerical semigroup $S$ is said to be modular with modulus $b$ and factor $a$ if $S=\{x \in \mathbb{N} \mid a x \bmod b \leq x\}$. The preceding remark ensures that $b-(a, b)-(a-1, b)$ is an invariant of $S$, which we call the weight of $S$ and denote by w $(S)$.

If $S$ is a numerical semigroup, the largest integer not in $S$ is called the Frobenius number of $S$ and is denoted by $\mathrm{g}(S)$. This integer has been widely studied; see for instance [Brauer 1942; Brauer and Shockley 1962; Johnson 1960; Selmer 1977; Sylvester 1884; Curtis 1990; Davison 1994; Djawadi and Hofmeister 1996]. In this direction it is worth highlighting [Ramírez Alfonsín 2000; $\geq 2005$ ], where a review of this problem is given, with many references. In the literature one can also find a large number of publications devoted to the study of one-dimensional analytically 
irreducible local domains via their value semigroups, which are numerical semigroups; see, for instance, [Apéry 1946; Barucci et al. 1997; Bertin and Carbonne 1977; Delorme 1976; Fröberg et al. 1987; Kunz 1970; Teissier 1973; Watanabe 1973]. As a consequence of this study, some interesting kinds of numerical semigroups arise, such as symmetric and pseudo-symmetric numerical semigroups. In Section 1 we prove that a modular numerical semigroup $S$ is symmetric if and only if $\mathrm{w}(S)=\mathrm{g}(S)$, and pseudo-symmetric if and only if $\mathrm{g}(S)=\mathrm{w}(S)+1$. Sections 3 and 4 are devoted to modular numerical semigroups with modulus equal to their weight plus two and three, respectively. We show that those of weight plus two are obtained from a symmetric numerical semigroup by adjoining its Frobenius number to it, and that those with weight plus three arise from a pseudo-symmetric numerical semigroup by adding to it its Frobenius number and this number divided by two.

In Section 5 we study those modular numerical semigroups $S$ such that the factor of $S$ divides the modulus. For these numerical semigroups we can explicitly give formulas for the multiplicity, the minimal generator set, the Apéry set and the Frobenius number, so the case $a \mid b$ is now well understood.

Section 6 addresses the problem of computing the Frobenius number in the complementary case $a \nmid b$, solving it when $(a-1)(a-(b \bmod a))<b$. We have not been able to solve the general case.

\section{Modular numerical semigroups}

Let $a$ and $b$ be integers such that $b \neq 0$. Since $a x \bmod b=(a \bmod b) x \bmod b$ and $a x \bmod b=a x \bmod (-b)$, in order to study the solutions of $a x \bmod b \leq x$, we can assume that $b$ is a positive integer and that $0 \leq a<b$.

Proposition 1. The set of integer solutions of a modular diophantine inequality is a numerical semigroup.

Proof. Let $a$ and $b$ be two integers such that $0 \leq a<b$ and let $S=\{x \in \mathbb{N} \mid$ $a x \bmod b \leq x\}$. Clearly $0 \in S$, and if $x$ is an integer greater than or equal to $b$, then $x \in S$. Hence $\mathbb{N} \backslash S$ is finite. For $x, y \in S$, we have $a(x+y) \bmod b \leq$ $a x \bmod b+a y \bmod b \leq x+y$, whence $x+y \in S$, so $S$ is closed under addition.

A numerical semigroup $S$ arising as in the proposition is said to be modular. The modular semigroup with modulus $b$ factor $a$ will be denoted by $\mathrm{S}(a, b)$; thus $\mathrm{S}(a, b)=\{x \in \mathbb{N} \mid a x \bmod b \leq x\}$. When we write $\mathrm{S}(a, b)$ we will generally assume tacitly that $a$ and $b$ are integers with $0 \leq a<b$.

Example 2. $\mathrm{S}(2,3)=\mathrm{S}(2,4)=\{0,2,3, \rightarrow\}$, where $\rightarrow$ means that all the elements beyond 3 are in the set. Thus $a$ and $b$ don't have to be unique. 
The goal of this section is to prove Theorem 12, which counts the natural numbers absent from $\mathrm{S}(a, b)$. We prepare the ground with some simple results.

Lemma 3. Let $a$ and $b$ be integers such that $0 \leq a<b$. Then $a x \bmod b \leq x$ if and only if $(b+1-a) x \bmod b \leq x$.

Proof. If $a x \bmod b \leq x$, there exist $q, r \in \mathbb{N}$ such that $a x=q b+r$ with $0 \leq r \leq x$. Hence $(b+1-a) x=(b+1) x-a x=b x-q b+x-r$ and $(b+1-a) x \bmod b \leq$ $x-r \leq x$. The converse follows by interchanging $a$ with $b+1-a$.

Lemma 4. Let $S$ be a modular numerical semigroup with modulus $b \geq 2$. Then there exists a positive integer a such that $a \leq \frac{1}{2}(b+1)$ and $S=\mathrm{S}(a, b)$.

Proof. Write $S=\mathrm{S}(a, b)$ with $0 \leq a<b$. By Lemma 3, $S=\mathrm{S}(b+1-a, b)$, so if $a>\frac{1}{2}(b+1)$ we can replace $a$ by $b+1-a \leq \frac{1}{2}(b+1)$. Also if $a=0$ we can replace it by $a=1$, since $S=\mathbb{N}$ for both these values of $a$.

Lemma 5. Let $a$ and $b$ be integers such that $0 \leq a<b$ and let $x \in \mathbb{N}$. Then

$$
a(b-x) \bmod b= \begin{cases}0 & \text { if } a x \bmod b=0, \\ b-(a x \bmod b) & \text { if } a x \bmod b \neq 0,\end{cases}
$$

and ax $\bmod b>x$ implies that $a(b-x) \bmod b<b-x$.

Corollary 6. If $S=\mathrm{S}(a, b)$ and $x \in \mathbb{N} \backslash S$, then $b-x \in S$.

Given a subset $A$ of $\mathbb{N}$, we denote by $\mathrm{H}(A)$ the complement $\mathbb{N} \backslash A$, and by $\langle A\rangle$ the submonoid of $\mathbb{N}$ generated by $A$ (the set of finite sums of elements of $A$ ).

Remark 7. If $S=\mathrm{S}(a, b) \neq \mathbb{N}$ for positive $a$ and $b$, then $b-1 \notin \mathrm{H}(S)$, since otherwise $b-(b-1)=1$ would be an element of $S$. Moreover $x \in S$ for all integers $x \geq b$. Therefore the Frobenius number $\mathrm{g}(S)$ is at most $b-2$.

We now characterize the case $\mathrm{g}(S)=b-2$. If $\mathrm{g}(S)=b-2$, Corollary 6 implies that $b-(b-2)=2 \in S$. Hence $b$ is odd and $S=\langle 2, b\rangle$. In addition, since $2 \in S$, $2 a \bmod b \leq 2$ and this leads to $2 a>b$, whence $a>\frac{1}{2} b$. But Lemma 4 says we can take $a \leq \frac{1}{2}(b+1)$, which then means $a=\frac{1}{2}(b+1)$. Hence $S=\mathrm{S}\left(\frac{1}{2}(b+1), b\right)$. Conversely, if $S=\mathrm{S}\left(\frac{1}{2}(b+1), b\right)$ with $b$ odd, it is easy to check that $S=\langle 2, b\rangle$ and thus $\mathrm{g}(S)=b-2$.

Example 8. Suppose $b \geq 2$ and $S=\mathrm{S}(2, b)$. Then $S=\left\{0,\left\lfloor\frac{1}{2}(b+1)\right\rfloor, \rightarrow\right\}$. For clearly $\{b, \rightarrow\} \subseteq S$. Now take $0<x<b$. Then $x \in S$ if and only if $2 x \bmod b \leq x$. However, $2 x \bmod b=2 x$ if and only if $2 x<b$, and thus in this case $x \notin S$. If $2 x \geq b$, then $2 x \bmod b=2 x-b \leq x$, whence $x \in S$.

Lemma 9. Let $S=\mathrm{S}(a, b)$ and let $x$ be an integer such that $0 \leq x \leq b$. Then $x$ and $b-x$ are both in $S$ if and only if $a x \bmod b \in\{0, x\}$. 
Proof. If $a x \bmod b \notin\{0, x\}$, Lemma 5 gives $a(b-x) \bmod b=b-(a x \bmod b)$. If $x \in S$, the right-hand side exceeds $b-x$ (since $a x \bmod b<x$ ). Thus $b-x \notin S$.

Conversely, if $a x \bmod b=0$, clearly $x \in S$ and also $b-x \in S$ by Lemma 5; whereas if $a x \bmod b=x \neq 0$, again $x \in S$, and Lemma 5 gives $a(b-x) \bmod b=$ $b-(a x \bmod b)=b-x$, so $b-x \in S$.

Lemma 10. Let $a$ and $b$ be positive integers and $x$ an integer such that $0 \leq x<b$.

(1) $a x \bmod b=0$ if and only if $x$ is a multiple of $b /(a, b)$.

(2) $a x \bmod b=x$ if and only if $x$ is a multiple of $b /(b, a-1)$.

Lemma 11. Let $S=S(a, b)$ with $0<a<b$. Let $\alpha=(b, a-1)$ and $\beta=(b, a)$, and let $x$ be an integer such that $0 \leq x \leq b$. Then

$$
\{x, b-x\} \subset S \Longleftrightarrow x \in\left\{0, \frac{b}{\alpha}, 2 \frac{b}{\alpha}, \ldots(\alpha-1) \frac{b}{\alpha}, \frac{b}{\beta}, 2 \frac{b}{\beta}, \ldots,(\beta-1) \frac{b}{\beta}, b\right\}=: X .
$$

The cardinality of $X$ is $\alpha+\beta$.

Proof. The equivalence is just Lemmas 9 and 10 put together. To show there is no duplication in the elements of $X$ as written, note that $(\alpha, \beta)=1$. If $s b / \alpha=t b / \beta$ for some $s, t \in \mathbb{N}$, then $s \beta=t \alpha=k \alpha \beta$ for some $k \in \mathbb{N}$. Hence $s=k \alpha$ and $t=k \beta$.

Theorem 12. Let $S=\mathrm{S}(a, b)$ for some integers $0 \leq a<b$. Then

$$
\# \mathrm{H}(S)=\frac{b+1-(a, b)-(a-1, b)}{2} .
$$

Here as usual \# denotes cardinality.

Proof. Let $\alpha, \beta$ and $X$ be as in Lemma 11. By Corollary 6 and Lemma 11, for $0 \leq x \leq b$, at most one of $x, b-x$ lies in $\mathrm{H}(S)$, and it's exactly one unless $x \in X$. Thus \# $\mathrm{H}(S)=\frac{1}{2}(b+1-\# X)=\frac{1}{2}(b+1-\alpha-\beta)$.

Example 13. If $p$ is an odd prime, $\# \mathrm{H}(\mathrm{S}(a, p))=\frac{1}{2}(p-1)$ for all $a$ with $1<a<p$.

As an immediate consequence of Theorem 12 we obtain:

Corollary 14. Suppose $\mathrm{S}(a, b)=\mathrm{S}(c, d)$. Then

$$
b-(a, b)-(a-1, b)=d-(c, d)-(c-1, d) .
$$

Example 15. The converse of Corollary 14 is false. For instance, $\langle 4,5,6\rangle=$ $\mathrm{S}(3,12) \neq \mathrm{S}(2,10)=\langle 5,6,7,8,9\rangle$.

Recall that we have defined the weight of $S=\mathrm{S}(a, b)$ as $\mathrm{w}(S):=b-(a, b)-$ $(a-1, b)$; by Theorem 12 , this number equals $2 \# \mathrm{H}(S)-1$, and so is an invariant of $S$. Note that $\mathrm{w}(\mathbb{N})=-1$. If $S \neq \mathbb{N}$, we can choose $a, b$ with $2 \leq a<b$; hence $(a, b)+(a-1, b) \leq \frac{1}{2} b+\frac{1}{3} b<b$, so $\mathrm{w}(S) \geq 1$. Thus, like the Frobenius number, the 
weight of a modular numerical semigroup is at least 1 , except for the case $S=\mathbb{N}$, where $\mathrm{w}(S)=\mathrm{g}(S)=-1$.

Theorem 12 and the inequality \# $H(S) \geq \frac{1}{2}(\mathrm{~g}(S)+1)$, valid for any numerical semigroup $S$ (see [Fröberg et al. 1987], for instance), yield:

Corollary 16. If $S$ is a modular numerical semigroup, then $\mathrm{w}(S)$ is odd and greater than or equal to $\mathrm{g}(S)$.

In view of this, modular numerical semigroups $S$ with w $(S)=\mathrm{g}(S)$ and $\mathrm{g}(S)$ odd, or with $\mathrm{w}(S)=\mathrm{g}(S)+1$ and $\mathrm{g}(S)$ even, have minimal possible weight with respect to their Frobenius numbers. The next result characterizes this kind of numerical semigroup, but before proving it we need to recall some concepts.

A numerical semigroup $S$ is symmetric if $x \in \mathbb{N} \backslash S$ implies $\mathrm{g}(S)-x \in S$. It is straightforward to prove that a symmetric numerical semigroup has odd Frobenius number. A numerical semigroup is pseudo-symmetric if $\mathrm{g}(S)$ is even and $x \in \mathbb{N} \backslash S$ implies that either $x=\mathrm{g}(S) / 2$ or $\mathrm{g}(S)-x \in S$. A numerical semigroup $S$ is symmetric if and only if \# $\mathrm{H}(S)=\frac{1}{2}(\mathrm{~g}(S)+1)$, and pseudo-symmetric if and only if \# $\left.\mathrm{H}(S)=\frac{1}{2}(\mathrm{~g}(S)+2)\right)$; see [Fröberg et al. 1987], for instance.

A numerical semigroup is irreducible if it cannot be expressed as the intersection of two numerical semigroups containing it properly. In [Rosales and Branco 2003] it is shown that $S$ is irreducible if and only if $S$ is symmetric or pseudo-symmetric (depending on the parity of $\mathrm{g}(S)$ ).

Corollary 17. Let $S$ be a modular numerical semigroup.

(1) $S$ is symmetric if and only if $\mathrm{w}(S)=\mathrm{g}(S)$.

(2) $S$ is pseudo-symmetric if and only if $\mathrm{w}(S)=\mathrm{g}(S)+1$.

Proof. $S$ is symmetric if and only if \# $\mathrm{H}(S)=\frac{1}{2}(\mathrm{~g}(S)+1)$. By Theorem 12, $\# \mathrm{H}(S)=\frac{1}{2}(\mathrm{w}(S)+1)$, whence $S$ is symmetric if and only if $\mathrm{g}(S)=\mathrm{w}(S)$. The proof of (2) is analogous.

Example 18. If $b$ is an odd integer, there exists a modular numerical semigroup $S$ with $\mathrm{w}(S)=b$. It suffices to take $S=\mathrm{S}(2, b+2)$, since $\mathrm{w}(\mathrm{S}(2, b+2))=b+2-$ $(2, b+2)-(1, b+2)=b+2-1-1=b$.

\section{Determining whether a numerical semigroup is modular}

In this section we give a procedure for deciding whether a given numerical semigroup is a modular numerical semigroup, and if so to express it in the form $\mathrm{S}(a, b)$.

Lemma 19. Let $S$ be a modular numerical semigroup with modulus $b$ and $S \neq \mathbb{N}$. Then $b \leq 12 \# \mathrm{H}(S)-6$.

Proof. As we saw right after Example 15, if $a \geq 2$ we have $(a, b)+(a-1, b) \leq \frac{5}{6} b$. By Theorem 12, \# $\mathrm{H}(S) \geq \frac{1}{2}\left(b+1-\frac{5}{6} b\right)$ and thus $b \leq 12 \# \mathrm{H}(S)-6$. 
For a numerical semigroup $S$, the multiplicity of $S$, denoted by $\mathrm{m}(S)$, is the least positive integer in $S$. Here is an immediate consequence of Lemma 11:

Lemma 20. For $S=\mathrm{S}(a, b)$,

$$
b-\mathrm{m}(S) \in S \Longleftrightarrow \mathrm{m}(S)=\min \left\{\frac{b}{(a, b)}, \frac{b}{(a-1, b)}\right\} .
$$

Lemma 21. Let $S$ be a modular numerical semigroup with modulus $b$. Then

$$
b \geq \mathrm{g}(S)+\mathrm{m}(S) .
$$

Proof. Since $1,2, \ldots, \mathrm{m}(S)-1$ are not in $S$, Corollary 6 ensures that $b-\mathrm{m}(S)+1$, $\ldots, b-1$ are. But $\{b, \mathrm{~m}(S)\} \subset S$, so $\{b-\mathrm{m}(S)+1, \rightarrow\} \subseteq S$. This implies that $\mathrm{g}(S) \leq b-\mathrm{m}(S)$.

Lemma 22. For $S=\mathrm{S}(a, b)$,

$$
b=\mathrm{g}(S)+\mathrm{m}(S) \Longleftrightarrow \mathrm{m}(S) \neq \min \left\{\frac{b}{(a, b)}, \frac{b}{(a-1, b)}\right\} .
$$

Proof. Follows from Lemmas 20 and 21.

Now we have all the ingredients to give the algorithm announced at the start of this section, to decide whether a numerical semigroup is of the form $S(a, b)$, and if so, produce such a pair $(a, b)$ (or all such pairs with $a \leq \frac{1}{2}(b+1)$, if the algorithm is not stopped after the first pair is found).

Algorithm 23. Given a numerical semigroup $S$ distinct from $\mathbb{N}$ :

(1) Compute \# $\mathrm{H}(S), \mathrm{g}(S)$ and $\mathrm{m}(S)$.

(2) Set $b=\mathrm{g}(S)+\mathrm{m}(S)$.

(3) For every $a \in A:=\left\{\begin{array}{l|l}a \in \mathbb{N} & \begin{array}{l}2 \leq a \leq \frac{1}{2}(b+1), \\ b=2 \# \mathrm{H}(S)+(a, b)+(a-1, b)-1, \\ \mathrm{~m}(S)<\min \{b /(a, b), b /(a-1, b)\}\end{array}\end{array}\right\}$ compute $\mathrm{S}(a, b)$; if $S=\mathrm{S}(a, b)$, return this answer and stop.

(4) Compute $B=\{b \in\{k \cdot \mathrm{m}(S) \mid k \in \mathbb{N}\} \mid 2 \# \mathrm{H}(S)+1 \leq b \leq 12 \# \mathrm{H}(S)-6\}$.

(5) For every $b \in B$

$$
\text { for every } a \in A_{b}:=\left\{\begin{array}{l|l}
a \in \mathbb{N} & \begin{array}{l}
2 \leq a \leq \frac{1}{2}(b+1), \\
b=2 \# \mathrm{H}(S)+(a, b)+(a-1, b)-1, \\
\mathrm{~m}(S)=\min \{b /(a, b), b /(a-1, b)\}
\end{array}
\end{array}\right\}
$$

compute $\mathrm{S}(a, b)$; if $S=\mathrm{S}(a, b)$, return this answer and stop.

(6) Return " $S$ is not modular". 
We briefly justify the correctness of Algorithm 23. In Steps (2) and (3) we check whether $S$ is a modular numerical semigroup with modulus $\mathrm{g}(S)+\mathrm{m}(S)$, and the correct working of these steps relies on Lemmas 4 and 22 and Theorem 12. If $S$ is not a modular numerical semigroup with modulus $\mathrm{g}(S)+\mathrm{m}(S)$, Lemma 22 gives $\mathrm{m}(S)=\min \{b /(a, b), b /(a-1, b)\}$. This implies that $\mathrm{m}(S)$ divides $b$. Theorem 12 states that $b=2 \# \mathrm{H}(S)+(a, b)+(a-1, b)-1$, so $b \geq 2 \# \mathrm{H}(S)+1$; at the same time $b \leq 12 \# \mathrm{H}(S)-6$ by Lemma 19. Therefore Steps (4) and (5) cover the case $b \neq \mathrm{g}(S)+\mathrm{m}(S)$.

Example 24. Let $S=\langle 3,5\rangle$. Then $\# \mathrm{H}(S)=4, \mathrm{~g}(S)=7$ and $\mathrm{m}(S)=3$. In Step (2) we get $b=10$. Step (3) yields $A=\{2,3,4\}$, then $S(2,10)=\langle 5,6,7,8,9\rangle$, $\mathrm{S}(3,10)=\langle 4,5,7\rangle$, and $\mathrm{S}(4,10)=\langle 3,5\rangle=S$, so the algorithm returns $S=$ $S(4,10)$.

Example 25. Let $S=\langle 3,8,10\rangle$. In this case \# $\mathrm{H}(S)=5, \mathrm{~g}(S)=7$ and $\mathrm{m}(S)=3$. In Step (2) we obtain $b=10$ and in Step (3), $A=\varnothing$. The only nonempty set $A_{b}$ with $b \in B$ is $A_{15}=\{5\}$. Since $S \neq \mathrm{S}(5,15)=\langle 3,7,11\rangle$, the algorithm returns No.

Example 26. Let $S=\langle 10,11,12\rangle$. Then $\# \mathrm{H}(S)=25, \mathrm{~g}(S)=49$ and $\mathrm{m}(S)=10$. In Step (2) we obtain $b=59$ and $A$ is empty. Computing $B$, we obtain

$B=\{60,70,80,90,100,110,120,130,140,150,160,170,180$,

$190,200,210,220,230,240,250,260,270,280,290\}$.

The only nonempty set $A_{b}$ with $b \in B$ is $A_{60}=\{6\}$. It turns out that $S=\mathrm{S}(6,60)$.

Remark 27. If the input to Algorithm 23 is known to be symmetric, the procedure can be improved, because if $S=\mathrm{S}(a, b)$ is symmetric then $b$ must be equal to $\mathrm{g}(S)+(a, b)+(a-1, b)$ (note that $\mathrm{w}(S)=\mathrm{g}(S)$ by Corollary 17). A similar argument applies to the pseudo-symmetric case.

Remark 28. The intersection $\bigcap_{i=1}^{n} \mathrm{~S}\left(a_{i}, b_{i}\right)$ of $n \geq 1$ modular numerical semigroups is a numerical semigroup; it need not be modular, as can be seen from Example 25, since we can write $\langle 3,8,10\rangle=\langle 3,4\rangle \cap\langle 3,5\rangle=\mathrm{S}(3,8) \cap \mathrm{S}(4,10)$.

Nor can every numerical semigroup be written as such an intersection: for instance, $\langle 7,8,10,13\rangle$ is a symmetric, hence irreducible, numerical semigroup; thus it cannot be an intersection of modular numerical semigroups other than by being itself a modular numerical semigroup. Algorithm 23 says that it is not.

\section{Modular numerical semigroups whose modulus is its weight plus two}

We now study modular numerical semigroups $S=\mathrm{S}(a, b)$ whose modulus $b$ equals $\mathrm{w}(S)+2$. Since $b=\mathrm{w}(S)+(a, b)+(a-1, b) \geq \mathrm{w}(S)+2$, the condition $b=\mathrm{w}(S)+2$ is equivalent to $(a, b)=(a-1, b)=1$ (so $b$ is odd), and it characterizes modular numerical semigroups whose moduli are minimal with respect to their weights. 
Every numerical semigroup $S$ is finitely generated (as an additive monoid). This is easy to see - for instance, start with two relatively prime $r, s \in S$ and then adjoin all elements of $S \cap\{0,1, \ldots, r s-1\}$ as yet unaccounted for. Among all generating sets one can of course choose one that is minimal, say $\mathcal{M}(S)$. A minute's thought shows that $M(S)$ is characterized by containing exactly those nonzero elements of $S$ that cannot be expressed as a sum of two nonzero elements of $S$ :

$$
\mathcal{M}(S)=(S \backslash\{0\}) \backslash((S \backslash\{0\})+(S \backslash\{0\})) .
$$

In particular, $\mathcal{M}(S)$ is unique. We set $\mathrm{e}(S)=\# \mathcal{M}(S)$ and call this number the embedding dimension of $S$; the elements of $\mathcal{M}(S)$ are called minimal generators.

Proposition 29. Let $S=\mathrm{S}(a, b)$ with $2 \leq a<b$ and $(a, b)=(a-1, b)=1$. Then

(1) $b=\mathrm{g}(S)+\mathrm{m}(S)$,

(2) $\# \mathrm{H}(S)=\frac{1}{2}(\mathrm{~g}(S)+\mathrm{m}(S)-1)$,

(3) $b$ is the largest minimal generator of $S$.

Proof. (1) We already know that $b-1 \in S$ when $2 \leq a<b$. Hence $\mathrm{m}(S) \neq b$. Using Lemma 22, we get $b=\mathrm{g}(S)+\mathrm{m}(S)$.

(2) Immediate from Theorem 12.

(3) First we prove that $b$ is a minimal generator of $S$. Assume to the contrary that $b=x+y$ with $x, y \in S \backslash\{0\}$. Then $a x \bmod b \leq x$ and $a y \bmod b \leq y$, and thus $(a x \bmod b)+(a y \bmod b) \leq x+y=b$. Since $a(x+y) \bmod b=a b \bmod b=0$, we deduce that $(a x \bmod b)+(a y \bmod b) \in\{0, b\}$. Thus either $a x \bmod b=x$ and $a y \bmod b=y$, or $a x \bmod b=0$ and $a y \bmod b=0$. These two cases contradict the two halves of Lemma 10.

To see that $b$ is the largest minimal generator, take $x \in S$ with $x>b$. By applying (1) we obtain $x>\mathrm{g}(S)+\mathrm{m}(S)$, which implies that $x-\mathrm{m}(S)>\mathrm{g}(S)$; this forces $x-\mathrm{m}(S) \in S$. Thus $x=\mathrm{m}(S)+(x-\mathrm{m}(S))$ cannot be a minimal generator of $S$.

Proposition 29 allows us to relate the modular numerical semigroups in question with unitary extensions of symmetric numerical semigroups or UESY-semigroups in short. A numerical semigroup $S$ is a UESY-semigroup if there exists a symmetric numerical semigroup $S^{\prime}$ such that $S^{\prime} \subset S$ and $\#\left(S \backslash S^{\prime}\right)=1$. In [Rosales $\geq 2005 \mathrm{~b}$ ] this condition is shown to be equivalent to the existence of a symmetric numerical semigroup $S^{\prime}$ such that $S=S^{\prime} \cup\left\{\mathrm{g}\left(S^{\prime}\right)\right\}$. The following result also appears there.

Proposition 30. Let $S$ be a numerical semigroup, $S \neq \mathbb{N}$. The following conditions are equivalent:

(1) $S$ is a UESY-semigroup.

(2) $\# \mathrm{H}(S)=\frac{1}{2}(\mathrm{~g}(S)+\mathrm{m}(S)-1)$ and $\mathrm{g}(S)+\mathrm{m}(S)$ is a minimal generator of $S$. $\square$ 
A pseudo-Frobenius number [Rosales and Branco 2002] of a numerical semigroup $S$ is an integer $x \notin S$ such that $x+s \in S$ for all $s \in S \backslash\{0\}$. The set of pseudoFrobenius numbers of $S$ is denoted by $\operatorname{Pg}(S)$, and its cardinality, called the type of $S$, is denoted by $\mathrm{t}(S)$. Clearly $\mathrm{g}(S) \in \operatorname{Pg}(S)$. Moreover $S$ is symmetric if and only if $\operatorname{Pg}(S)=\{\mathrm{g}(S)\}$, and $S$ is pseudo-symmetric if and only if $\operatorname{Pg}(S)=\left\{\mathrm{g}(S), \frac{1}{2} \mathrm{~g}(S)\right\}$; see [Barucci et al. 1997; Fröberg et al. 1987], for instance.

In [Rosales $\geq 2005 \mathrm{~b}$ ] it is proved that if $S$ is a UESY-semigroup distinct from $\mathbb{N}$, then $\mathrm{t}(S)=\mathrm{e}(S)-1$. This, plus Propositions 29 and 30, gives:

Corollary 31. Let $S=\mathrm{S}(a, b)$ be such that $2 \leq a<b$ and $(a, b)=(a-1, b)=1$. Then $\mathrm{t}(S)=\mathrm{e}(S)-1$ and there exists a symmetric numerical semigroup $S^{\prime}$ such that $S=S^{\prime} \cup\left\{\mathrm{g}\left(S^{\prime}\right)\right\}$.

Theorem 32. Let $S=\mathrm{S}(a, b)$. Then $b=\mathrm{w}(S)+2$ if and only if $S$ is a UESYsemigroup and $b$ is a minimal generator of $S$.

Proof. If $b=\mathrm{w}(S)+2=b-(a, b)-(a-1, b)+2$, we deduce $(a, b)=(a-1, b)=1$. Corollary 31 then says that $S$ is a UESY-semigroup, and Proposition 29 that $b$ is a minimal generator of $S$.

Conversely, if $b$ is a minimal generator of $S$ it equals $\mathrm{g}(S)+\mathrm{m}(S)$, by Lemma 21 and the fact, shown in the proof of Proposition 29, that a minimal generator of $S$ cannot exceed $\mathrm{g}(S)+\mathrm{m}(S)$. If $S$ is a UESY, then, \# $\mathrm{H}(S)=\frac{1}{2}(\mathrm{~g}(S)+m(S)-1)$ by Proposition 30 and \# $\mathrm{H}(S)=\frac{1}{2}(\mathrm{w}(S)+1)$ by Theorem 12 . Thus $b=\mathrm{w}(S)+2$.

Corollary 33. Let $S$ be a modular numerical semigroup with modulus $b$. Then $b=\mathrm{w}(S)+2$ if and only if $S \backslash\{b\}$ is a symmetric numerical semigroup. Therefore, if $b$ is a prime integer, $S \backslash\{b\}$ is a symmetric numerical semigroup.

Proof. If $b=\mathrm{w}(S)+2$, Theorem 32 says $b$ is a minimal generator of $S$, so $S^{\prime}=S \backslash\{b\}$ is a numerical semigroup with $\mathrm{g}\left(S^{\prime}\right)=b$. By Corollary $6, S^{\prime}$ is symmetric.

Conversely, if $S \backslash\{b\}$ is a symmetric numerical semigroup, then $S$ is a UESYsemigroup with $b$ as a minimal generator. Now Theorem 32 gives $b=\mathrm{w}(S)+2$.

Finally, $b$ prime implies $(a, b)=(a-1, b)=1$, so $\mathrm{w}(S)=b-2$.

Corollary 34. Let $b \geq 3$ be an integer. Then $b$ is prime if and only if $b$ is the largest minimal generator of $\mathrm{S}(a, b)$ for every a such that $2 \leq a \leq \sqrt{b}$.

Proof. If $b$ is prime Proposition 29 applies; this proves one direction. Conversely, suppose $b$ is not a prime - say $b=a c$ with integers $a, c \geq 2$ and $a \leq \sqrt{b}$. For $S=\mathrm{S}(a, b)$, we have $a c \bmod b=0$ and thus $c \in S$. But then $b=a c$ cannot be a minimal generator of $S$.

\section{Modular numerical semigroups whose modulus is its weight plus three}

We now study modular numerical semigroups $S=\mathrm{S}(a, b)$ such that $b=\mathrm{w}(S)+3$; this condition is equivalent to $(a, b)+(a-1, b)=3$. There are two cases: 
- $(a, b)=1$ and $(a-1, b)=2$.

- $(a, b)=2$ and $(a-1, b)=1$.

In both situations $b$ must be even and by Corollary 6 we deduce that $\frac{1}{2} b \in S$.

Let $S$ be a numerical semigroup with minimal generating set $\left\{n_{1}, \ldots, n_{p}\right\}$. We say that $x \in S$ has a unique expression if the equality $x=a_{1} n_{1}+\cdots+a_{p} n_{p}$, with $a_{1}, \ldots, a_{p} \in \mathbb{N}$, determines $a_{1}, \ldots, a_{p}$ uniquely.

Proposition 35. Let $S=\mathrm{S}(a, b)$ be such that $2 \leq a<b$ and $(a, b)+(a-1, b)=3$.

(1) $\mathrm{m}(S) \neq \frac{1}{2} b \Leftrightarrow S \neq\left\{0, \frac{1}{2} b, \rightarrow\right\} \Leftrightarrow b=\mathrm{g}(S)+\mathrm{m}(S) \Leftrightarrow \# \mathrm{H}(S)=\frac{\mathrm{g}(S)+\mathrm{m}(S)-2}{2}$.

(2) $\frac{1}{2} b$ is a minimal generator of $S$.

(3) $b$ has a unique expression in $S$.

Proof. (1) Follows easily from Corollary 6, Lemma 22 and Theorem 12.

(2) Suppose $x+y=\frac{1}{2} b$ with $x, y \in S$. Then $a x \bmod b \leq x$ and $a y \bmod b \leq y$, whence $a x \bmod b+a y \bmod b \leq x+y=\frac{1}{2} b$. Thus $\frac{1}{2} a b \bmod b=a(x+y) \bmod b=$ $a x \bmod b+a y \bmod b$. We must show that $x=0$ or $y=0$. We distinguish two cases. If $(a, b)=2$, then $\frac{1}{2} a b \bmod b=0$, so $a x \bmod b=0$ and $a y \bmod b=0$; then Lemma 10 shows that both $x$ and $y$ are multiples of $\frac{1}{2} b$, which leads to the desired conclusion. Similarly, if $(a-1, b)=2$, then $\frac{1}{2} a b \bmod b=\frac{1}{2} b$, so $a x \bmod b=x$ and $a y \bmod b=y$; Lemma 10 again shows that $x$ and $y$ are multiples of $\frac{1}{2} b$.

(3) We prove that if $x, y \in S \backslash\{0\}$ are such that $x+y=b$, then $x=y=\frac{1}{2} b$. Arguing as in the proof of Proposition 29(3), we see that either $(a x \bmod b$, ay $\bmod y)=(x, y)$ or $a x \bmod b=a y \bmod y=0$. Lemma 10 implies that $x$ and $y$ are both multiples of $\frac{1}{2} b$, and since $x \neq 0 \neq y$, we conclude that $x=y=\frac{1}{2} b$.

Paralleling what we did in Section 3 for the case $b=\mathrm{w}(S)+2$, we can use Proposition 35 to relate modular numerical semigroups such that $b=\mathrm{w}(S)+3$ with a previous studied class of numerical semigroups. A numerical semigroup $S$ is called a PESPY-semigroup if there exists a pseudo-symmetric numerical semigroup $S^{\prime}$ such that $S=S^{\prime} \cup\left\{\frac{1}{2} \mathrm{~g}\left(S^{\prime}\right), \mathrm{g}\left(S^{\prime}\right)\right\}$ (the two additional elements are the pseudoFrobenius numbers of $S^{\prime}$; see [Barucci et al. 1997; Fröberg et al. 1987]).

Numerical semigroups of the form $\{0, x, \rightarrow\}$ with $x$ a positive integer are called intervals. The following result appears in [Rosales $\geq 2005 \mathrm{a}$ ].

Proposition 36. Let $S$ be a numerical semigroup that is not an interval. The following conditions are equivalent:

(1) $S$ is a PEPSY-semigroup.

(2) $\# \mathrm{H}(S)=\frac{1}{2}(\mathrm{~g}(S)+\mathrm{m}(S)-2), \frac{1}{2}(\mathrm{~g}(S)+\mathrm{m}(S))$ is a minimal generator of $S$ and $\mathrm{g}(S)+\mathrm{m}(S)$ is an element of unique expression of $S$. 
The next result is an immediate consequence of Propositions 35 and 36.

Corollary 37. Let $S=\mathrm{S}(a, b)$ be such that $2 \leq a<b,(a, b)+(a-1, b)=3$ and $S$ is not an interval. Then $S$ is a PEPSY-semigroup.

In [Rosales $\geq 2005$ a] it is proved that if $S$ is a PEPSY-semigroup that is not an interval, then $\mathrm{t}(S)=\mathrm{e}(S)-1$. Thus:

Corollary 38. Let $S=\mathrm{S}(a, b)$ be such that $2 \leq a<b,(a, b)+(a-1, b)=3$ and $S$ is not an interval. Then $\mathrm{t}(S)=\mathrm{e}(S)-1$.

Remark 39. Among numerical semigroups, interval semigroups have maximal embedding dimension relative to multiplicity: $\mathrm{e}(S)=\mathrm{m}(S)$. For any numerical semigroup with maximal embedding dimension, $\mathrm{t}(S)=\mathrm{m}(S)-1=\mathrm{e}(S)-1$ (see [Barucci et al. 1997], for instance). Hence the assumption " $S$ is not an interval" can be dropped from Corollary 38 .

Theorem 40. Assume that $S=\mathrm{S}(a, b)$ is not an interval. Then $b=\mathrm{w}(S)+3$ if and only if $S$ is a PEPSY-semigroup, $\frac{1}{2} b$ is a minimal generator of $S$ and $b$ has $a$ unique expression in $S$.

Proof. Necessity follows from Corollary 37 and Proposition 35 . Sufficiency: Lemma 21 says that $b \geq \mathrm{g}(S)+\mathrm{m}(S)$. If $b>\mathrm{g}(S)+\mathrm{m}(S)$, then $\mathrm{m}(S)+(b-\mathrm{m}(S))$ and $\frac{1}{2} b+\frac{1}{2} b$ are distinct expressions for $b$ in $S\left(\mathrm{~m}(S) \neq \frac{1}{2} b\right.$ since otherwise $S$ is an interval, by Corollary 6). Therefore $b=\mathrm{g}(S)+\mathrm{m}(S)$. By Proposition 36, we know that $\# \mathrm{H}(S)=\frac{1}{2}(\mathrm{~g}(S)+\mathrm{m}(S)-2)$ and Theorem 12 ensures that $\# \mathrm{H}(S)=\frac{1}{2}(\mathrm{w}(S)+1)$, whence $b=\mathrm{g}(S)+\mathrm{m}(S)=\mathrm{w}(S)+3$.

Corollary 41. Let $S$ be a modular numerical semigroup with modulus $b$. Then $b=\mathrm{w}(S)+3$ if and only if $S \backslash\left\{\frac{1}{2} b, b\right\}$ is a pseudo-symmetric numerical semigroup. Therefore, if $b=2 p$ and $a<p$ for some positive prime $p$, then $S \backslash\left\{\frac{1}{2} b, b\right\}$ is a pseudo-symmetric numerical semigroup.

Proof. Suppose $b=\mathrm{w}(S)+3$. By Theorem $40, \frac{1}{2} b$ is a minimal generator of $S$ and $b$ has a unique expression in $S$. This implies that $S^{\prime}=S \backslash\left\{\frac{1}{2} b, b\right\}$ is a numerical semigroup, and clearly $\mathrm{g}\left(S^{\prime}\right)=b$. Using Corollary 6 we can easily deduce that $S^{\prime}$ is pseudo-symmetric.

Conversely, if $S \backslash\left\{\frac{1}{2} b, b\right\}$ is a pseudo-symmetric numerical semigroup, then $S$ is a PEPSY-semigroup by definition, $\frac{1}{2} b$ is a minimal generator of $S$ and $b=\frac{1}{2} b+\frac{1}{2} b$ is the unique expression of $b$ in $S$. Thus $b=\mathrm{w}(S)+3$ by Theorem 40 .

\section{When the factor divides the modulus}

We next focus on numerical semigroups of the form $S=\mathrm{S}(a, a b)$, where we may as well assume $a, b>1$. First a general definition: given a numerical semigroup 
$S$ and $n \in S \backslash\{0\}$, the Apéry set of $n$ in $S$ [Apéry 1946] is

$$
\operatorname{Ap}(S, n)=\{s \in S \mid s-n \notin S\} .
$$

This set always has $n$ elements $w(0)=0, w(1), \ldots, w(n-1)$, where $w(i)$ is the least element congruent to $i$ modulo $n$. Note also that $x \in \mathbb{Z}$ is an element of $S$ if and only if $x \geq w(x \bmod n)$. Consequently

$$
\mathrm{g}(S)=\max (\operatorname{Ap}(S, n))-n .
$$

The following result is a consequence of [Rosales 1996, Lemma 3.3] and gives a characterization of Apéry sets which will be useful later.

Lemma 42. Let $m>0$ be an integer and let $X=\{0=w(0), w(1), \ldots, w(m-1)\}$ be a subset of $\mathbb{N}$ such that $i<w(i) \equiv i \bmod m$ for all $i \in\{1, \ldots, m-1\}$. Let $S$ be the submonoid of $\mathbb{N}$ generated by $X \cup\{m\}$. Then $S$ is a numerical semigroup with multiplicity $m$. Moreover, $\operatorname{Ap}(S, m)=X$ if and only if for all $i, j \in\{1, \ldots, m-1\}$ there exist $k \in\{0, \ldots, m-1\}$ and $t \in \mathbb{N}$ such that $w(i)+w(j)=w(k)+t m$.

Getting back to $S=\mathrm{S}(a, a b)$, with $a, b>1$, we will give a description of the particular Apéry set $\operatorname{Ap}(S, \mathrm{~m}(S))$ in terms of $a, b$, and this will lead to an explicit formula for the Frobenius number of $S$. We also show how the minimal generating set for such numerical semigroups can be computed from $a$ and $b$ as well as the corresponding sets of pseudo-Frobenius numbers.

Lemma 43. $\mathrm{m}(\mathrm{S}(a, a b))=b$.

Proof. Let $S=\mathrm{S}(a, a b)$ and let $x \in\{1, \ldots, b-1\}$. Then $a x<a b$ and thus $a x \bmod$ $a b=a x>x$, whence $x \notin S$. Clearly $b \in S$ and consequently $\mathrm{m}(S)=b$.

Theorem 44. $\operatorname{Ap}(\mathrm{S}(a, a b), b)=\left\{0, k_{1} b+1, k_{2} b+2, \ldots, k_{b-1} b+b-1\right\}$, where $k_{i}=\lceil(a-1) i / b\rceil$ for all $i \in\{1, \ldots, b-1\}$.

Proof. Let $S^{\prime}$ be the semigroup generated by $\left\{b, k_{1} b+1, \ldots, k_{b-1} b+b-1\right\}$. Since $k_{i} \geq 1$ for all $i \in\{1, \ldots, b-1\}$ we have $\mathrm{m}\left(S^{\prime}\right)=b$. Clearly $k_{1} \leq \cdots \leq k_{b-1}$ and $k_{i}+k_{j} \geq k_{i+j}$ for all $i, j \in\{1, \ldots, b-1\}$ with $2 \leq i+j \leq b-1$. Using Lemma 42 , we deduce that $\operatorname{Ap}\left(S^{\prime}, b\right)=\left\{0, k_{1} b+1, \ldots, k_{b-1} b+b-1\right\}$. Recall that $x \in \mathbb{Z}$ belongs to $S^{\prime}$ if and only if $x \geq k_{x} \bmod b b+x \bmod b$, since this latter number is the element in $\operatorname{Ap}\left(S^{\prime}, b\right)$ that is congruent to $x$ modulo $b$. So, for $x$ an integer we have $x \in S^{\prime} \Longleftrightarrow\lfloor x / b\rfloor \geq k_{x} \bmod b \Longleftrightarrow\lfloor x / b\rfloor \geq\lceil(a-1)(x \bmod b) / b\rceil \Longleftrightarrow$ $\lfloor x / b\rfloor \geq(a-1)(x \bmod b) / b \Longleftrightarrow\lfloor x / b\rfloor b \geq(a-1)(x \bmod b) \Longleftrightarrow x-(x \bmod b) \geq$ $(a-1)(x \bmod b) \Longleftrightarrow a(x \bmod b) \leq x \Longleftrightarrow a x \bmod a b \leq x$. Thus $S^{\prime}=\mathrm{S}(a, a b)$.

Using this result and equality $(*)$ with $n=\mathrm{m}(S)$, we obtain:

Corollary 45. $\mathrm{g}(\mathrm{S}(a, a b))=\lceil(b-1)(a-1) / b\rceil b-1$. 
Particularizing the formula given in Theorem 12 for the case at hand, we get

$$
\# \mathrm{H}(\mathrm{S}(a, a b))=\frac{a(b-1)-(a-1, b)+1}{2} .
$$

Minimal generators. We next turn our attention to the minimal generating set $\left\{n_{0}<n_{1}<\cdots<n_{p}\right\}$ of $\mathrm{S}(a, a b)$. We know that $n_{0}=b$, by Lemma 43; our goal is to describe the remaining minimal generators.

Lemma 46. Let $x$ and $y$ be positive integers. Then $\lceil x / b\rceil+\lceil y / b\rceil=\lceil(x+y) / b\rceil$ if and only if $x \equiv 0 \bmod b$ or $y \equiv 0 \bmod b$ or $(x \bmod b)+(y \bmod b)>b$.

Remark 47. If $S$ is any numerical semigroup and $m \in S \backslash\{0\}$, then $S$ is generated by $X=(\operatorname{Ap}(S, m) \backslash\{0\}) \cup\{m\}=\{m, w(1), \ldots, w(m-1)\}$, and the minimal generating set of $S$ is $X \backslash(X+X)$. Now, in the case of $S=\mathrm{S}(a, a b)$, Theorem 44 says that $\operatorname{Ap}(S, b)=\left\{0, k_{1} b+1, \ldots, a k_{b-1} b+b-1\right\}$, with $k_{i}=\lceil(a-1) i / b\rceil$ for all $i \in\{1, \ldots, b-1\}$. Thus $k_{t} b+t$ is a minimal generator of $S$ if and only if $k_{t} \neq k_{i}+k_{t-i}$ for all $i \in\{1, \ldots, t-1\}$.

Lemma 48. Let $S=\mathrm{S}(a, a b)$ with $a, b>1$, set $k_{i}=\lceil(a-1) i / b\rceil$ for all $i \in$ $\{1, \ldots, b-1\}$ and take $t \in\{1, \ldots, b-1\}$.

(i) If $t<b /(a-1, b)$, then $k_{t} b+t$ is a minimal generator of $S$ if and only if $(a-1) i \bmod b<(a-1) t \bmod b$ for all $i \in\{1, \ldots, t-1\}$.

(ii) If $t>b /(a-1, b)$, then $k_{t} b+t$ is not a minimal generator of $S$.

(iii) If $t=b /(a-1, b)$, then $k_{t} b+t$ is a minimal generator of $S$.

Proof. Using Lemma 46 and Remark 47, we see that $k_{t} b+t$ is a minimal generator of $S$ if and only if $(a-1) i \not \equiv 0 \bmod b$ and $(a-1) i \bmod b+(a-1)(t-i) \bmod b \leq b$ for all $i \in\{1, \ldots, t-1\}$. Observe that

$$
\frac{b}{(a-1, b)}=\frac{\operatorname{lcm}(a-1, b)}{a-1}=\min \{i \mid(a-1) i \bmod b=0\} .
$$

(i) From the foregoing we deduce that if $t<b /(a-1, b)$, then $k_{t} b+t$ is a minimal generator of $S$ if and only if $(a-1) i \bmod b+(a-1)(t-i) \bmod b \leq b$ for all $i \in$ $\{1, \ldots, t-1\}$. If $(a-1) i \bmod b+(a-1)(t-i) \bmod b=b$, then $(a-1) t \bmod b=0$, which is impossible in view of $(\dagger)$, since $t<b /(a-1, b)$. Hence $k_{t} b+t$ is a minimal generator of $S$ if and only if for all $i \in\{1, \ldots, t-1\}$ one has $(a-1) i \bmod$ $b+(a-1)(t-i) \bmod b<b$, which is equivalent to $(a-1) i \bmod b+(a-1)(t-$ $i) \bmod b=(a-1) t \bmod b$. Since $(a-1)(t-i) \bmod b \neq 0$, we conclude that $k_{t} b+t$ is a minimal generator of $S$ if and only if $(a-1) i \bmod b<(a-1) t \bmod b$ for all $i \in\{1, \ldots, t-1\}$.

(ii) Let $i=b /(a-1, b)$. Then $(a-1) i \equiv 0 \bmod b$ and in view of Lemma 46 we get $k_{i}+k_{t-i}=k_{t}$, which implies that $k_{t} b+b$ is not a minimal generator of $S$. 
(iii) In this setting $(a-1) t \bmod b=0$ and $(a-1) i \bmod b \neq 0$ for all $i \in\{1, \ldots, t-1\}$. Hence for every $i \in\{1, \ldots, t-1\}$ one gets $(a-1) i \bmod b+(a-1)(t-i) \bmod b=b$, and by Lemma 46 we deduce that $k_{t} \neq k_{i}+k_{t-i}$ for any $i \in\{1, \ldots, t-1\}$. Therefore $k_{t} b+t$ is a minimal generator of $S$.

Lemma 48 yields an explicit description of the minimal generating set of $S$ :

Theorem 49. Let $S=\mathrm{S}(a, a b)$ with $a, b>1$, and set $k_{i}=\lceil(a-1) i / b\rceil$ for $i \in$ $\{1, \ldots, b-1\}$.

(1) If $(b, a-1)=1$, the minimal generating set of $S$ is $\left\{b, k_{t_{1}} b+t_{1}, \ldots, k_{t_{r}} b+t_{r}\right\}$, where $\left\{t_{1}, \ldots, t_{r}\right\}=\{t \in\{1, \ldots, b-1\} \mid(a-1) i \bmod b<(a-1) t \bmod b$ for all $i \in\{1, \ldots, t-1\}\}$.

(2) If $(b, a-1) \neq 1$, let $t_{r+1}=b /(b, a-1)$. Then the minimal generating set of $S$ is $\left\{b, k_{t_{1}} b+t_{1}, \ldots, k_{t_{r}} b+t_{r}, k_{t_{r+1}} b+t_{r+1}\right\}$, where $\left\{t_{1}, \ldots, t_{r}\right\}=\{t \in$ $\left\{1, \ldots, t_{r+1}-1\right\} \mid(a-1) i \bmod b<(a-1) t \bmod b$ for all $\left.i \in\{1, \ldots, t-1\}\right\}$.

Example 50. Let $S=\mathrm{S}(5,35)$. Applying Theorem 49(1) with $a=5$ and $b=7$, we see that $\left\{t_{1}, \ldots, t_{r}\right\}=\{1,3,5\}$ (observe that 1 is always in $\left\{t_{1}, \ldots, t_{r}\right\}$ ), and that $S$ is minimally generated by $\{7,8,17,26\}$.

Example 51. Let $S=\mathrm{S}(5,30)$. Applying Theorem 49(2) with $a=5$ and $b=6$, we see that $t_{r+1}=3,\left\{t_{1}, \ldots, t_{r}\right\}=\{1\}$, and $S$ is minimally generated by $\{6,7,15\}$.

Corollary 52. Let $S=\mathrm{S}(a, a b)$ with $a, b>1$. Set $k_{i}=\lceil(a-1) i / b\rceil$ for $i \in$ $\{1, \ldots, b-1\}$, and

$$
t= \begin{cases}\min \{x \in \mathbb{N} \mid(a-1) x \equiv b-1 \bmod b\} & \text { if }(b, a-1)=1, \\ b /(b, a-1) & \text { if }(b, a-1) \neq 1 .\end{cases}
$$

Then $k_{t} b+t$ is the greatest minimal generator of $S$.

Corollary 53. Let $a \geq 3$ and let $b$ be a positive integer. Then $\mathrm{e}(\mathrm{S}(a, a b)) \geq$ $\lfloor b /(a-1)\rfloor+1$.

Proof. The integer $b$ is always a minimal generator of $\mathrm{S}(a, a b)$. Also, if $(a-1) t \leq b$, then by Lemma $48, k_{t} b+t$ is a minimal generator of $S$.

Pseudo-Frobenius numbers. For any numerical semigroup $S$, we define an order $\leq_{S}$ on $S$ as follows: $a \leq_{S} b$ if $b-a \in S$. Given a subset $A$ of $S$, denote by $\operatorname{Max}_{{ }_{S}} A$ the set of maximal elements of $A$ with respect to $\leq_{s}$. The following result appears in [Rosales and Branco 2002].

Lemma 54. Let $S$ be any numerical semigroup with multiplicity $m$. If

$$
\operatorname{Max}_{\leq S}(\operatorname{Ap}(S, m))=\left\{w_{i_{1}}, \ldots, w_{i_{t}}\right\}
$$

the pseudo-Frobenius numbers of $S$ (page 387) are precisely $w_{i_{1}}-m, \ldots, w_{i_{t}}-m$. 
Note that if $w, w^{\prime} \in \operatorname{Ap}(S, m)$ and $w-w^{\prime} \in S$, this forces $w-w^{\prime}$ to be in $\operatorname{Ap}(S, m)$ as well. Hence

$\operatorname{Max}_{\leq S}(\operatorname{Ap}(S, m))$

$$
=\left\{w \in \operatorname{Ap}(S, m) \mid w+w^{\prime} \notin \operatorname{Ap}(S, m) \text { for all } 0 \neq w^{\prime} \in \operatorname{Ap}(S, m)\right\} .
$$

Let $S=\mathrm{S}(a, a b)$ with $a, b>1$. Our aim is to compute the set $\operatorname{Max}_{\leq S}(\operatorname{Ap}(S, b))$ and thus, in view of Lemma 54, the pseudo-Frobenius set $\operatorname{Pg}(S)$.

Remark 55. By Theorem 44, $k_{i} b+i \notin \operatorname{Max}_{\leq_{S}}(\operatorname{Ap}(S, b))$ if and only if there exists $j \in\{1, \ldots, b-1\}$ such that $i+j \leq b-1$ and $k_{i}+k_{j}=k_{i+j}$. Minimal generators are $\leq s$-minimal elements of $\operatorname{Ap}(S, b)$, which is why the condition just stated is similar (dual) to the one presented on the previous page for minimal generators.

Theorem 56. Let $a$ and $b$ be two integers greater than one, and let $S=\mathrm{S}(a, a b)$. Let $k_{i}=\lceil(a-1) i / b\rceil$ for $i \in\{1, \ldots, b-1\}$. Then $k_{i} b+i \in \operatorname{Max}_{\leq s}(\operatorname{Ap}(S, b))$ if and only if one of the following conditions hold:

(i) $(a-1) i \equiv 0 \bmod b$ and $i=b-1$,

(ii) $(a-1) i \not \equiv 0 \bmod b$ and for all $t \in\{i+1, \ldots, b-1\}$, either $(a-1) i \bmod b<$ $(a-1) t \bmod b$ or $(a-1) t \bmod b=0$.

Proof. Assume that $(a-1) i \equiv 0 \bmod b$ and $i<b-1$. Then by Lemma 46, we deduce that $k_{i}+k_{1}=k_{i+1}$ and thus $k_{i} b+i \notin \operatorname{Max}_{\leq S}(\operatorname{Ap}(S, b))$. If $(a-1) i \not \equiv$ $0 \bmod b$, then by Lemma 46 we have $k_{i} b_{i}+i \in \operatorname{Max}_{\leq S}(\operatorname{Ap}(S, b))$ if and only if for all $t \in\{i+1, \ldots, b-1\}$ we have $(a-1)(t-i) \not \equiv 0 \bmod b$ and $(a-1) i \bmod$ $b+(a-1)(t-i) \bmod b \leq b$. If $(a-1) i \bmod b+(a-1)(t-i) \bmod b<b$, then $(a-1) i \bmod b+(a-1)(t-i) \bmod b=(a-1) t \bmod b$ and thus $(a-1) i \bmod b<$ $(a-1) t \bmod b$. If $(a-1) i \bmod b+(a-1)(t-i) \bmod b=b$, then $(a-1) t \bmod b=0$.

To prove the converse, assume $k_{i} b+i \notin \operatorname{Max}_{\leq s}(\operatorname{Ap}(S, b))$. Then there exists $t \in\{1+i, \ldots, b-1\}$ such that $k_{i}+k_{t-i}=k_{t}$. By using Lemma 46, we deduce that $(a-1) i \equiv 0 \bmod b$ or $(a-1)(t-i) \equiv 0 \bmod b$ or $(a-1) i \bmod b+(a-1)(t-i) \bmod$ $b>b$. If $(a-1) i \equiv 0 \bmod b$, then $i$ must be equal to $b-1$, but this is impossible since $t \in\{i+1, \ldots, b-1\}$. If $(a-1)(t-i) \equiv 0 \bmod b$, then $(a-1) i \bmod b=$ $(a-1) t \bmod b$, which is also impossible by hypothesis. Finally if $(a-1) i \bmod b+$ $(a-1)(t-i) \bmod b>b$, then $(a-1) t \bmod b=(a-1) i \bmod b+(a-1)(t-i) \bmod$ $b-b<(a-1) i \bmod b$, leading again to a contradiction.

Example 57. Let $S=\mathrm{S}(5,30)$. Applying Theorem 56 we get $\operatorname{Max}_{\leq S}(\operatorname{Ap}(S, 6))=$ $\{29\}$, which by Lemma 54 means that $\operatorname{Pg}(S)=\{23\}$. Thus $S(5,30)$ is symmetric.

Proposition 58. Let $S=\mathrm{S}(a, a b)$ with $a, b>1$.

(1) $S$ is symmetric if and only if $(a-1, b)+(a-1) \bmod b=b$.

(2) $S$ is pseudo-symmetric if and only if $(a-1, b)+(a-1) \bmod b=b+1$. 
Proof. (1) Combining Corollaries 45 and 17(1), we see that $S$ is symmetric if and only if $\lceil(b-1)(a-1) / b\rceil b-1=a b-a-(a-1, b)$. The left-hand side can be written as $(a-1-\lfloor(a-1) / b\rfloor) b-1=(a-1) b-\lfloor(a-1) / b\rfloor b-1=a b-b-(a-1-$ $(a-1) \bmod b)-1$. Thus $S$ is symmetric if and only if $(a-1) \bmod b+(a-1, b)=b$.

(2) As above, but this time using Corollary 17(2).

Corollary 59. Let $k$ be a positive integer and let $b$ be a multiple of $k$. Then $\mathrm{S}(b-k+1+b n,(b-k+1+b n) b)$ is symmetric for all $n \in \mathbb{N}$.

The pseudo-symmetric case is completely different:

Corollary 60. S(a,ab) is not pseudo-symmetric for any choice of $a, b>1$.

Proof. Set $q=\lfloor(a-1) / b\rfloor$ and choose $u, v \in \mathbb{Z}$ such that $(a-1, b)=u(a-1)+v b$. If $\mathrm{S}(a, a b)$ is pseudo-symmetric, we have $(a-1, b)+(a-1) \bmod b=b+1$, hence $u(a-1)+v b+(a-1)-q b=b+1$, or yet $(u+1)(a-1)+(v-q-1) b=1$. But this implies $(a-1, b)=1$ and hence $1+(a-1) \bmod b=b+1$, an impossibility.

Some families. We now present some families of numerical semigroups of the form $\mathrm{S}(a, a b)$ with $a, b>1$ such that $(a-1, b)=1$. For these families we can compute the minimal generating set and pseudo-Frobenius numbers explicitly. As a consequence of Theorems 49 and 56 one gets:

Proposition 61. Let $S=\mathrm{S}(a, a b)$ with $a, b>1$ and $(a-1, b)=1$. Set $k_{i}=$ $\lceil(a-1) i / b\rceil$ for $i \in\{1, \ldots, b-1\}$ and take $t \in\{1, \ldots, b-1\}$.

(1) $k_{t} b+t$ is a minimal generator of $S$ if and only if $(a-1) i \bmod b<(a-1) t \bmod$ $b$ for all $i \in\{1, \ldots, t-1\}$.

(2) $k_{t} b+t \in \operatorname{Max}_{\leq s}(\operatorname{Ap}(S, b))$ if and only if $(a-1) t \bmod b<(a-1) i \bmod b$ for all $i \in\{t+1, \ldots, b-1\}$.

Let $\mathrm{S}_{n}$ be the symmetric group in $n$ elements $\{1, \ldots, n\}$, and for $k$ relatively prime to $n+1$, define the permutation $\sigma_{k, n+1} \in \mathrm{S}_{n}$ by $\sigma(i)=k i \bmod (n+1)$ for $i=1, \ldots, n$. Such a permutation is called modular. Next, given any permutation $\sigma \in \mathrm{S}_{n}$, set

$$
\begin{aligned}
& \mathrm{E}(\sigma)=\{t \in\{1, \ldots, n\} \mid \sigma(i)<\sigma(t) \text { for all } i \in\{1, \ldots, t-1\}\}, \\
& \mathrm{T}(\sigma)=\{t \in\{1, \ldots, n\} \mid \sigma(t)<\sigma(i) \text { for all } i \in\{t+1, \ldots, n\}\} .
\end{aligned}
$$

With this notation we can rewrite Proposition 61 as follows.

Corollary 62. Let $S=\mathrm{S}(a, a b)$ with $a, b>1$ and $(a-1, b)=1$. Then

$$
\mathrm{e}(S)=\# \mathrm{E}\left(\sigma_{a-1, b}\right)+1 \text { and } \mathrm{t}(S)=\# \mathrm{~T}\left(\sigma_{a-1, b}\right) .
$$

The minimal generating set of $S$ is $\{b\} \cup\left\{\lceil(a-1) i / b\rceil b+i \mid i \in \mathrm{E}\left(\sigma_{a-1, b}\right)\right\}$, and

$$
\operatorname{Max}_{\leq S}(\operatorname{Ap}(S, b))=\left\{\lceil(a-1) i / b\rceil b+i \mid i \in \mathrm{T}\left(\sigma_{a-1, b}\right)\right\} .
$$


Example 63. Let $S=\mathrm{S}(6,42)$. Apply Corollary 62 with $a=6$ and $b=7$. Clearly $\sigma_{5,7}=(154623), \mathrm{E}\left(\sigma_{5,7}\right)=\{1,4\}$ and $\mathrm{T}\left(\sigma_{5,7}\right)=\{3,6\}$. Hence $\mathrm{e}(S)=3$ and $\mathrm{t}(S)=2$. The set $\{7,\lceil(5 \times 1) / 7\rceil 7+1,\lceil(5 \times 4) / 7\rceil 7+4\}=\{7,8,25\}$ is a minimal generating set of $S$ and $\operatorname{Max}_{\leq S}(\operatorname{Ap}(S, 7))=\{\lceil(5 \times 3) / 7\rceil 7+3,\lceil(5 \times 6) / 7\rceil 7+6\}=\{24,41\}$.

Corollary 64. Let $S=\mathrm{S}((b-1)+b n,((b-1)+b n) b)$ with $n \in \mathbb{N}$ and $b \geq 5$ odd. Then $S$ is minimally generated by $\left\{b,(n+1) b+1,\left(\frac{b-1}{2}+n \frac{b+1}{2}\right) b+\frac{b+1}{2}\right\}$, and

$$
\operatorname{Max}_{\leq S}(\operatorname{Ap}(S, b))=\left\{\left(\frac{b-1}{2}+n \frac{b-1}{2}\right) b+\frac{b-1}{2},((b-2)+n(b-1)) b+b-1\right\} .
$$

Proof. Since $(b-2+b n, b)=(b-2, b)=1$, we can apply Corollary 62. By inspection we see that $\mathrm{E}\left(\sigma_{b-2, b}\right)=\{1,(b+1) / 2\}$ and $\mathrm{T}\left(\sigma_{b-2, b}\right)=\{(b-1) / 2, b-1\}$. We can conclude the proof using Corollary 62, taking into account that

$$
\begin{gathered}
\left\lceil\frac{((b-2)+b n) 1}{b}\right\rceil=n+1, \quad\left\lceil\frac{((b-2)+b n)(b \pm 1) / 2}{b}\right\rceil=\frac{b-1}{2}+n \frac{b \pm 1}{2}, \quad \text { and } \\
\left\lceil\frac{((b-2)+b n)(b-1)}{b}\right\rceil=(b-2)+n(b-1) .
\end{gathered}
$$

Corollary 65. Let $b$ be an integer greater than or equal to two and let $n \in \mathbb{N}$. Then $S=\mathrm{S}\left((n+1) b,(n+1) b^{2}\right)$ is minimally generated by $\{b,(n+1) b+1\}$ and $\operatorname{Max}_{\leq S}(\operatorname{Ap}(S, b))=\{(n+1)(b-1) b+b-1\}$.

Proof. Use Corollary 62 and the fact that $\sigma_{(n+1) b-1, b}=\sigma_{b-1, b}$ swaps $i$ and $b-i$.

Corollary 66. Let $S=\mathrm{S}(2+n b,(2+n b) b)$ with $n \in \mathbb{N}$ and $b \geq 2$. Then $S$ is minimally generated by

$$
X=\{b,(n+1) b+1,(2 n+1) b+2, \ldots,((b-1) n+1) b+b-1\}
$$

and $\operatorname{Max}_{\leq S}(\operatorname{Ap}(S, b))=X \backslash\{b\}$.

Proof. Use Corollary 62 and the fact that $\sigma_{1+n b, b}=\sigma_{1, b}$ is the identity.

Corollary 67. Let $S=\mathrm{S}(3+n b,(3+n b) b)$ with and $n \in \mathbb{N} b \geq 3$ odd. Then $S$ is minimally generated by $\left\{b,(n+1) b+1,(2 n+1) b+2, \ldots,\left(\frac{b-1}{2} n+1\right)+\frac{b-1}{2}\right\}$ and

$$
\operatorname{Max}_{\leq S}(\operatorname{Ap}(S, b))=\left\{\left(\frac{b+1}{2} n+2\right) b+\frac{b+1}{2}, \ldots,((b-1) n+2) b+b-1\right\} .
$$

Proof. By considering $\sigma_{2+b n, b}=\sigma_{2, b}$ we see that $\mathrm{E}\left(\sigma_{2, b}\right)=\left\{1, \ldots, \frac{1}{2}(b-1)\right\}$ and $\mathrm{T}\left(\sigma_{2, b}\right)=\left\{\frac{1}{2}(b+1), \ldots, b-1\right\}$. Using Corollary 62 , the proof follows easily from

$$
\left\lceil\frac{(2+b n) i}{b}\right\rceil b= \begin{cases}(n i+1) b+i & \text { if } i \leq \frac{1}{2}(b-1), \\ (n i+2) b+i & \text { if } i \geq \frac{1}{2}(b+1) .\end{cases}
$$




\section{The Frobenius number in other special cases}

In Section 5 we studied $\mathrm{S}(a, b)$ with $a \mid b$. We now give some partial results for the Frobenius number in the complementary case, $a \nmid b$. We are able to find the number when $(a-1)(a-(b \bmod a))<b$. We use without further comment the fact that, for $q$ a rational number and $x$ a positive integer, $x<\lceil q\rceil$ implies $x<q$.

Lemma 68. Let $S=\mathrm{S}(a, b)$ with $0<a<b$ and $b \bmod a \neq 0$. Then

$$
\mathrm{g}(\mathrm{S}(a, b)) \leq b-\lceil b / a\rceil .
$$

Proof. Let $x$ be a positive integer. If $x<\lceil b / a\rceil$, then $x<b / a$ and thus $a x \bmod b=$ $a x>x$. Hence $x \notin S$ and in view of Corollary 6, this leads to $b-x \in S$. As $y \in S$ for all $y \geq b$, we conclude that $\mathrm{g}(S) \leq b-\lceil b / a\rceil$.

Lemma 69. Let $a$ and $b$ be positive integers such that $a<b$ and $b \bmod a \neq 0$. Then $a\lceil b / a\rceil \bmod b=a-(b \bmod a)$.

Proposition 70. Let $a$ and $b$ be positive integers such that $a<b$ and $b \bmod a \neq 0$. Then $\mathrm{g}(\mathrm{S}(a, b))=b-\lceil b / a\rceil$ if and only if $(a-1)(a-(b \bmod a))<b$.

Proof. Let $S=\mathrm{S}(a, b)$. From Lemma 68 we deduce that $\mathrm{g}(S)=b-\lceil b / a\rceil$ if and only if $b-\lceil b / a\rceil \notin S$, or in other words, $a(b-\lceil b / a\rceil) \bmod b>b-\lceil b / a\rceil$. This by Lemma 69 is equivalent to $((b \bmod a)-a) \bmod b>b-\lceil b / a\rceil$, and this condition holds if and only if $b+(b \bmod a)-a>b-\lfloor b / a\rfloor-1$. Hence $\operatorname{g}(S)=b-\lceil b / a\rceil$ if and only if $\lfloor b / a\rfloor+1+(b \bmod a)>a$, or equivalently $(b-(b \bmod a)) / a+1+$ $(b \bmod a)>a$, and this holds if and only if $b>(a-1)(a-(b \bmod a))$.

Corollary 71. Let $a$ and $b$ be positive integers such that $a<b, b \bmod a \neq 0$ and $(a-1)(a-(b \bmod a))<b$. Then $\mathrm{m}(\mathrm{S}(a, b))=\lceil b / a\rceil$.

Proof. Let $S=\mathrm{S}(a, b)$. By Proposition 70, we know that $\mathrm{g}(S)=b-\lceil b / a\rceil$. Thus $b-\lceil b / a\rceil \notin S$ and thus by Corollary $6,\lceil b / a\rceil=b-(b-\lceil b / a\rceil) \in S$. Besides, if $x$ is a positive integer such that $x<\lceil b / a\rceil$, then $x<b / a$, whence $a x \bmod b=a x>x$ and thus $x \notin S$. Therefore $\mathrm{m}(S)=\lceil b / a\rceil$.

Though we have given an explicit formula for $\mathrm{g}(\mathrm{S}(a, b))$ for several cases, we have not been able to find such a formula for arbitrary positive integers $a$ and $b$. We propose this as an open question.

Problem 1. Find a formula for $\mathrm{g}(\mathrm{S}(a, b))$ with $a$ and $b$ positive integers.

\section{Acknowledgments}

We thank J. I. García-García and the referee for their comments and suggestions. 


\section{References}

[Apéry 1946] R. Apéry, "Sur les branches superlinéaires des courbes algébriques", C. R. Acad. Sci. Paris 222 (1946), 1198-1200. MR 8,221a Zbl 0061.35404

[Barucci et al. 1997] V. Barucci, D. E. Dobbs, and M. Fontana, Maximality properties in numerical semigroups and applications to one-dimensional analytically irreducible local domains, Mem. Amer. Math. Soc. 598, Amer. Math. Soc., Providence, 1997. MR 97g:13039 Zbl 0868.13003

[Bertin and Carbonne 1977] J. Bertin and P. Carbonne, "Semi-groupes d'entiers et application aux branches", J. Algebra 49:1 (1977), 81-95. MR 58 \#27957 Zbl 0498.14016

[Brauer 1942] A. Brauer, "On a problem of partitions", American J. Math. 64 (1942), 299-312. MR 3,270d Zbl 0061.06801

[Brauer and Shockley 1962] A. Brauer and J. E. Shockley, "On a problem of Frobenius", J. Reine Angew. Math. 211 (1962), 215-220. MR 26 \#6113 Zbl 0108.04604

[Curtis 1990] F. Curtis, "On formulas for the Frobenius number of a numerical semigroup", Math. Scand. 67:2 (1990), 190-192. MR 92e:11019 Zbl 0734.11009

[Davison 1994] J. L. Davison, "On the linear Diophantine problem of Frobenius", J. Number Theory 48:3 (1994), 353-363. MR 95j:11033 Zbl 0805.11025

[Delorme 1976] C. Delorme, "Sous-monoïdes d'intersection complète de N.", Ann. Sci. École Norm. Sup. (4) 9:1 (1976), 145-154. MR 53 \#10821 Zbl 0325.20065

[Djawadi and Hofmeister 1996] M. Djawadi and G. Hofmeister, "Linear Diophantine problems", Arch. Math. (Basel) 66:1 (1996), 19-29. MR 96j:11029 Zbl 0854.11016

[Fröberg et al. 1987] R. Fröberg, C. Gottlieb, and R. Häggkvist, “On numerical semigroups”, Semigroup Forum 35:1 (1987), 63-83. MR 88d:20092 Zbl 0614.10046

[Johnson 1960] S. M. Johnson, “A linear diophantine problem”, Canad. J. Math. 12 (1960), 390398. MR 22 \#12074 Zbl 0096.02803

[Kunz 1970] E. Kunz, "The value-semigroup of a one-dimensional Gorenstein ring”, Proc. Amer. Math. Soc. 25 (1970), 748-751. MR 42 \#263 Zbl 0197.31401

[Ramírez Alfonsín 2000] J. L. Ramírez Alfonsín, “The Diophantine Frobenius problem”, preprint 00893, Forschungsinstitut für Diskrete Mathematik, Bonn, 2000.

[Ramírez Alfonsín $\geq 2005]$ J. L. Ramírez Alfonsín, "The Diophantine Frobenius problem". In preparation.

[Rosales 1996] J. C. Rosales, “On numerical semigroups”, Semigroup Forum 52:3 (1996), 307-318. MR 96m:20092 Zbl 0853.20041

[Rosales $\geq 2005$ a] J. C. Rosales, "Adding or removing an element from a pseudo-symmetric numerical semigroup", preprint.

[Rosales $\geq 2005 b$ ] J. C. Rosales, "Numerical semigroups that differ from a symmetric numerical semigroup in one element", to appear in Algebra Colloquium.

[Rosales and Branco 2002] J. C. Rosales and M. B. Branco, "Numerical semigroups that can be expressed as an intersection of symmetric numerical semigroups", J. Pure Appl. Algebra 171:2-3 (2002), 303-314. MR 2003b:20089 Zbl 1006.20043

[Rosales and Branco 2003] J. C. Rosales and M. B. Branco, "Irreducible numerical semigroups", Pacific J. Math. 209:1 (2003), 131-143. MR 2004b:20091 Zbl 02101065

[Selmer 1977] E. S. Selmer, "On the linear Diophantine problem of Frobenius”, J. Reine Angew. Math. 293/294 (1977), 1-17. MR 56 \#246 Zbl 0349.10009 
[Sylvester 1884] J. Sylvester, "Mathematical questions with their solutions", Educational Times 41 (1884), 21.

[Teissier 1973] B. Teissier, "Appendice" in Le problème des modules pour les branches planes, École Polytechnique, Paris, 1973. MR 54 \#2662 Zbl 0317.14004

[Watanabe 1973] K. Watanabe, "Some examples of one dimensional Gorenstein domains", Nagoya Math. J. 49 (1973), 101-109. MR 47 \#6689 Zbl 0257.13024

Received March 20, 2003. Revised March 16, 2004.

\author{
J. C. Rosales \\ DEPARTAMENTo DE Álgebra \\ UNIVERSIDAD DE GRANADA \\ E-18071 GRANADA \\ SPAIN \\ jrosales@ugr.es \\ P. A. GARCÍA-SÁNCHEZ \\ Departamento de Álgebra \\ UNIVERSIDAD DE GRANADA \\ E-18071 GRANADA \\ SPAIN \\ pedro@ugr.es \\ J. M. URBANO-BLANCO \\ DEPARTAMEnto de Álgebra \\ UNIVERSIDAD DE GRANADA \\ E-18071 GRANADA \\ SPAIN
}

jurbano@ugr.es 